\title{
METHOD OF STATISTICAL CONTROL OF THE SUPPLY RELIABILITY
}

\author{
P. E. Issouribehere*, J. C. Barbero*, J. L. Martínez** and A. Galinski**
}

(*) IITREE-LAT, Argentina; (**) ENRE, Argentina

\begin{abstract}
In Buenos Aires metropolitan area, electrical energy distribution must comply with maximum supply interruption rates, imposed on distributors by Franchise Agreements.

The calculation procedure of the corresponding discounts (bonus) includes the record of network contingencies, type of customer-network connection and non-supplied energy estimation.

The control method which is described, comprises the record of interruptions at supply points and the following calculation of the corresponding bonus.

By selecting these points with statistical sampling criteria, it is possible to determine the quality of the Utility global task performed by traditional methods. This method is similar to that one used to control electrical energy meters in service.

The present starting-up stage of the method provides deterministic information about long term interruptions obtained from records of supply interruptions, carried out for about three years of systematic recording.

Also recorded short interruptions (durations comprised between 10 sec. and 3 minutes) are analyzed. At present, these short interruptions are not penalized by the Argentine regulation, but this topic has increasing importance to qualify supply reliability to industries.
\end{abstract}

Keywords: Power Quality - Long term Interruption Control - Statistics.

\section{SUPPLIED ENERGY VS. NON-SUPPLIED ENERGY CONCEPT (INTERRUPTIONS).}

The electric energy supplied to a customer is billed by reading the meter and multiplying this value by an appropriate rate. As it is established in the Private Utility's Franchise Agreements of Buenos Aires metropolitan area, the control on the whole energy meters in-service must be achieved by statistical sampling techniques. These controls are already taking place and they allow to establish if the Utilities meet a statistically allowable percentage of bad meters.

On the other hand, contracts impose to Utilities the corresponding discounts on the customer's bills when maximum supply interruption rates and duration are not fulfilled. By these rules, it can be said that a customer pays to the Utility for the energy supplied, and is paid by the Utility for the non-supplied energy. A graphical scenario is shown in Fig.1

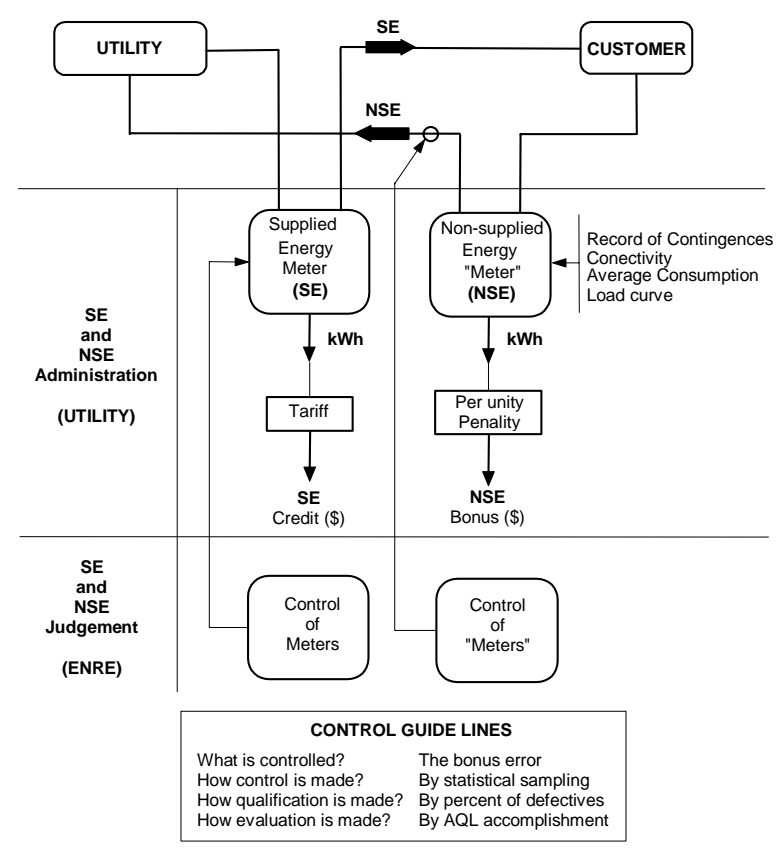

Fig. 1. Technical judgement of the Supplied Energy (SE) and Non-supplied Energy (NSE) Measurement.

Thus, it would seem reasonable to measure both energies with equal rules.

Since there are not non-supplied energy (NSE) meters of massive diffusion at present in the market, the Contracts have anticipated the calculation of that magnitude, by relating the contingencies of the network with each customer, and by determining the NSE based on the time and duration of each interruption, on the average consumption and on a universal load curve.

The NSE determination should be made with a "class" according to the supplied energy (SE) measurement. Furthermore, if the whole in-service meters are checked by statistical sampling, the same control procedure could be applied for the NSE measurement.

\section{PROPOSED METHOD FOR THE CONTROL OF THE DISCOUNT (BONUS) BY NSE.}

The used method is based on the direct control over points at customer supply level, selected on the basis of statistical sampling techniques. 
This method takes into account the frequency of interruptions and the accumulated duration of interruptions per semester.

1) $n$ customers per Utility will be controlled.

2) In the period of interest the bonus (discount due to bad quality) for each of the $n$ customers inspected $\left(b_{r}\right)$ will be calculated.

3) In the same period, the error $(e)$ in the bonus calculated by the Utility $\left(b_{d}\right)$, by means of the traditional method, will be as follows:

$$
e=\frac{b_{d}-b_{r}}{b_{r}} \cdot 100 \quad[\%]
$$

4) The allowable $c$ error limit to calculate the bonus that the Utility must apply will be defined. Thus by comparing the $e$ error with the allowable $c$ limit, it may result that:

$\checkmark \quad|e| \leq c \quad$ : calculated values by the Utility have been correct.

$\diamond \quad e<c<0$ : calculated values have not been taken into account by the Utility either all the interruptions or their true duration, or both.

$\checkmark \quad e>c>0$ : calculated values by the Utility have been incorrect, deducing more than what corresponds.

5) The performance of the Utility in this task will be statistically evaluated.

If $n$ is adequately selected according to the total number of customers of the Utility, it is possible to obtain a coefficient that reflects effectiveness to trace the supply interruptions. Such a coefficient would be the AQL (acceptable quality level) which is used to qualify the quality of great lots of meters, evaluated by sampling.

\section{INTERRUPTIONS IN THE BUENOS AIRES METROPOLITAN AREA.}

At present electrical distribution in Buenos Aires metropolitan area must comply with maximum interruption rates for supply interruptions longer than 3 minutes, imposed to the Utilities in the Franchise Agreements (1).

The interruption administration system employed by the Utilities is based on queries performed over both, the interruption data base and the data base which describes the Customer-Network connectivity. Transgressions to the decided limits give rise to discounts (bonus) to each user.

The control method applied today by the Regulatory Agency ENRE (2) includes interruption recording at supply points (customer level).

In this work that information recorded is used to obtain some indicative figures of the present situation concerning short and long interruptions. Putting into practice the proposed method of statistical evaluation will be deferred for the immediate future.

\section{Employed Event Recorders and Data Processed.}

The employed event recorders detect the following types of interruptions in the supply network:

$\checkmark$ microinterruption 1: short interruptions, up to $1 \mathrm{~s}$ long.

$\checkmark$ microinterruption 2: short interruptions, $1 \mathrm{~s}$ to $10 \mathrm{~s}$ long.

$\checkmark$ microinterruption 3: short interruptions, $10 \mathrm{~s}$ to 3 minutes long.

$\checkmark$ Interruptions: long interruptions (duration longer than 3 minutes)

The main object of the recorders installation is to detect interruptions on MV lines or higher voltage levels on the network. To reach this objective the recorders are installed in couples, each one in a location (customer address) connected to the LV network and both located on the same MV line. In this way interruptions on the monitored MV line or higher voltage levels, will be detected and recorded as coincident events in the installed recorders.

In this work the information obtained at customer level is employed to make microinterruption and interruption statistics.

All the information processed comprises the period October 1997 to June 2000 for the three Utilities of the Buenos Aires metropolitan area (here after named A, B and $\mathrm{C}$ ).

Table 1 presents indicative numbers of the processed data and its representativeness.

Table 1.- Details of the sampling made.

\begin{tabular}{|c|c|c|c|}
\hline Utility & Stations & Locations & Month/Location \\
\hline A & 26 & 486 & 5.18 \\
\hline B & 26 & 492 & 5.19 \\
\hline C & 9 & 388 & 5.11 \\
\hline
\end{tabular}

Processing Results.

Statistical processing was oriented to obtain indexes expressed as average values by monitored location per semester, for the four mentioned interruption categories. The processing target was to obtain some Electrical Service Quality indicators about short interruptions and some indicative figures of the present situation that can be compared with the levels published by UNIPEDE (3) as a guide of the European Standard EN 50160 application and with the values established in the Franchise Agreements (1).

Criteria to identify and suppress interruptions caused by customers (such as holidays, weekends, daily and periodic interruptions) were defined to minimize errors in statistics. 
The following processing was made for each Utility.

For each monitored location the following magnitudes were obtained.

$$
\begin{aligned}
& D_{\text {tot }}=\sum_{i=1}^{N_{\text {tot }}} D_{i} \\
& \bar{D}=\frac{1}{N_{\text {tot }}} \sum_{i=1}^{N_{\text {tot }}} D_{i} \\
& \lambda=6 \frac{N_{\text {tot }}}{M i} \\
& r=\frac{\bar{D}}{10 \mathrm{Mi}} \\
& Q=\frac{D_{\text {tot }}}{10 \mathrm{Mi}}
\end{aligned}
$$

Being for each location:

- $D_{i}$ : Interruption duration [min].

- $N_{\text {tot }}$ : Total number of recorded interruptions.

- Mi : Number of months that the location was monitored.

- $\lambda$ : Interruption rate expressed in [interruptions / semester]

- $r$ : Mean time to repair expressed in [hours / interruption].

- Q : Unavailability [hours / semester]

Figures 1 to 3 show statistic results on a semester basis for the interruption rate $(\lambda)$, the mean time to repair $(r)$ and the Unavailability $(Q)$.

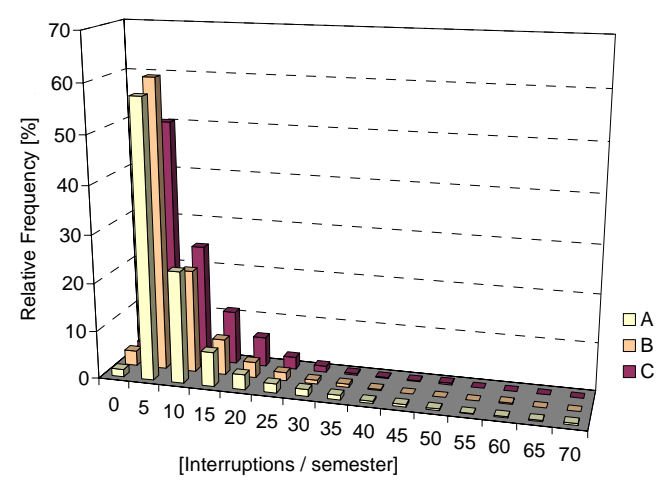

(a)

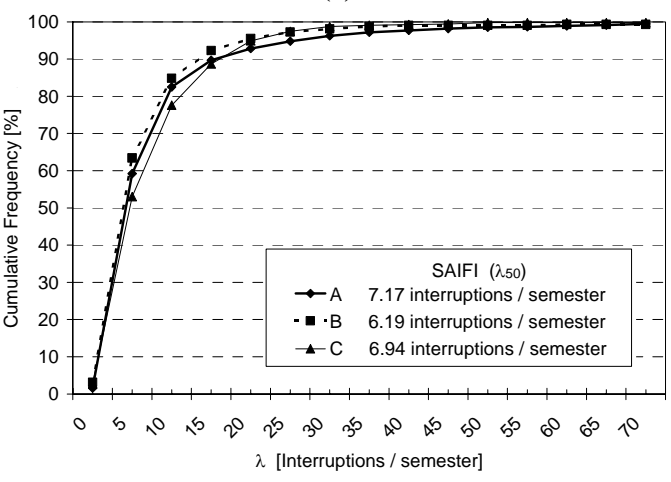

(b)

Fig-1. Interruption rate $(\lambda)$. (a) Relative frequency histograms. (b) Cumulative frequency curves. Average values should be compared with the SAIFI which must be obtained from the overall interruption administration.

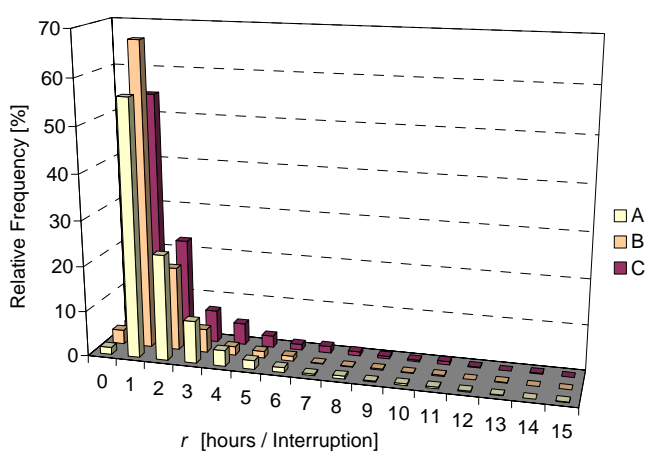

(a)

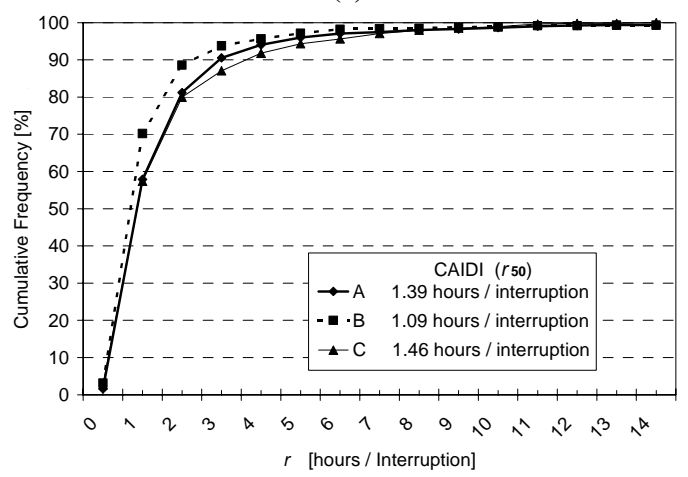

(b)

Fig-2. Mean time to repair ( $r$ ). (a) Relative frequency histograms. (b) Cumulative frequency curves. Average values should be compared with the CAIDI.

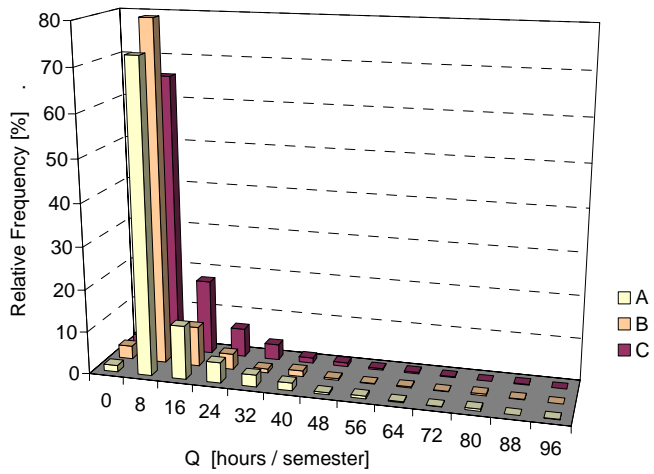

(a)

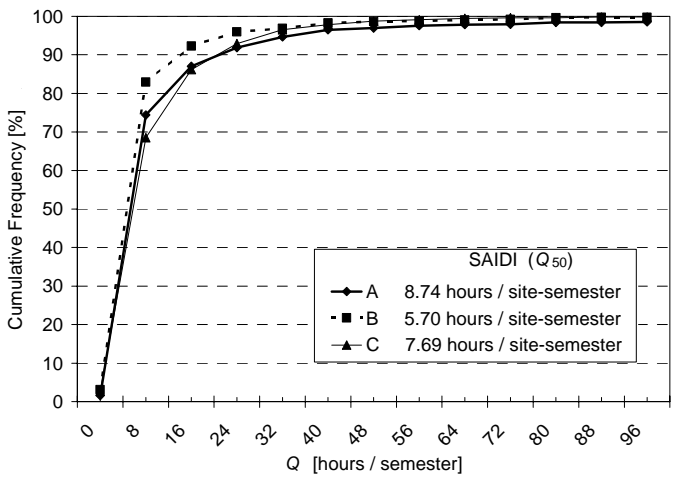

(b)

Fig-3. Unavailability (Q). (a) Relative frequency histograms. (b) Cumulative frequency curves. Average values should be compared with the SAIDI. 
Average values of the corresponding index $(\lambda, r$ and $Q)$ for each Utility are also indicated. These values should be compared respectively with the SAIFI (System Average Interruption Frequency Index, which must be obtained from the administration of the overall interruption occurred in the network), CAIDI (Customer Average Interruption Duration Index) and with the SAIDI (System Average Interruption Duration Index).

Short interruptions up to 1 sec. long. Obtained information is partial since employed monitors do not record interruptions of $100 \mathrm{~ms}$ or shorter duration. The information is slanted, that is why it does not appear here.

Short interruptions 1 sec. to $10 \mathrm{sec}$. long. Figure 4 shows the relative frequency histograms and the cumulative frequency curves for the number of microinterruptions recorded by location-semester.

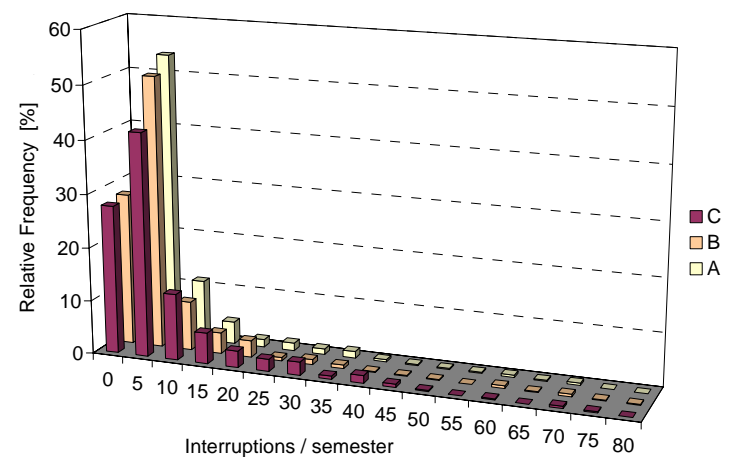

(a)

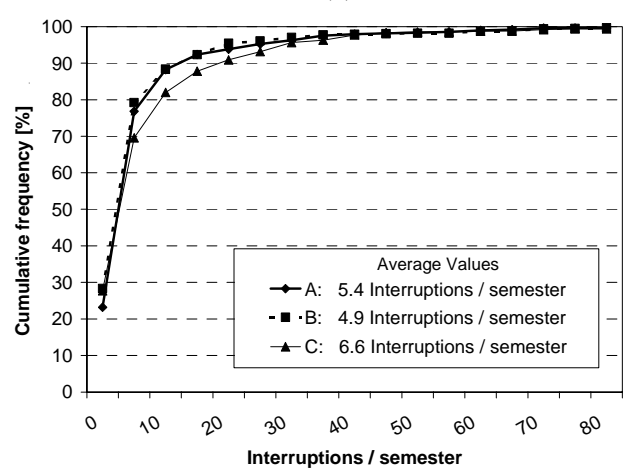

(b)

Fig-4. Microinterruption rate (durations between $1 \mathrm{~s}$ and $10 \mathrm{~s}$ ). (a) Relative frequency histograms. (b) Cumulative frequency curves. Values with $50 \%$ probability (statistical mean) have been adopted as microinterruption rate (by location-semester).

Short interruptions 10 sec. to 3 min. long. Figure 5 shows the relative frequency histograms and the cumulative frequency curves for the number of microinterruptions recorded by location-semester.

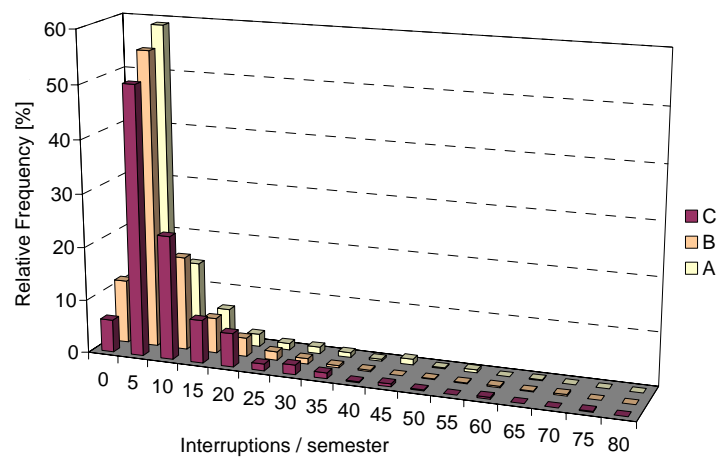

(a)

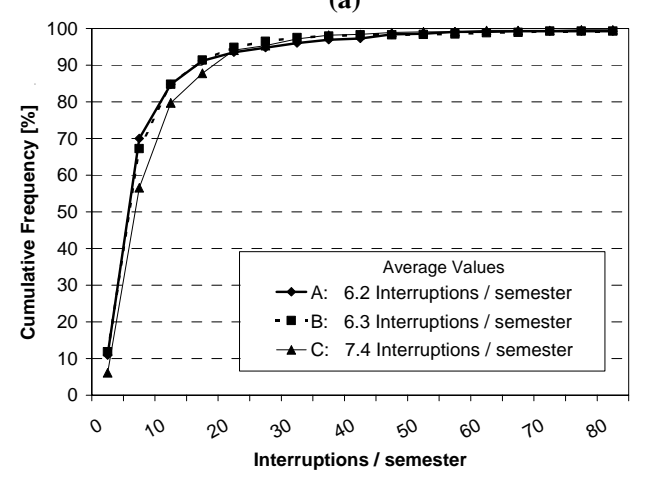

(b)

Fig-5. Microinterruption rate (durations between $10 \mathrm{~s}$ and $3 \mathrm{~min}$ ). (a) Relative frequency histograms. (b) Cumulative frequency curves. Values with $50 \%$ probability (statistical mean) have been adopted as microinterruption rate (by location-semester).

Figure 6 shows analysis results of available data about microinterruption frequencies as a function of microinterruption duration.

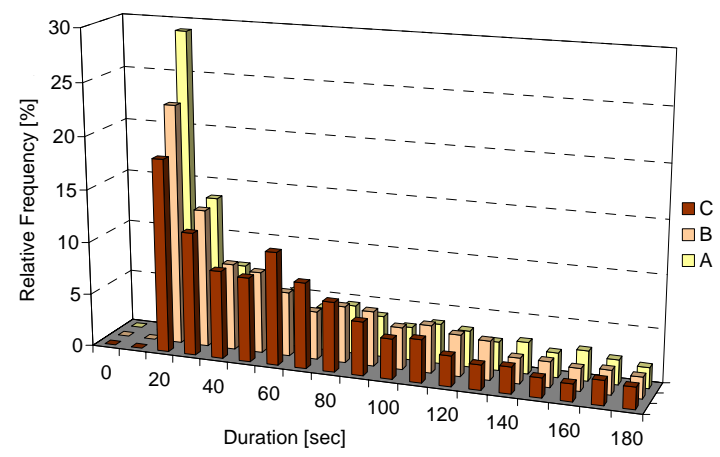

(a)

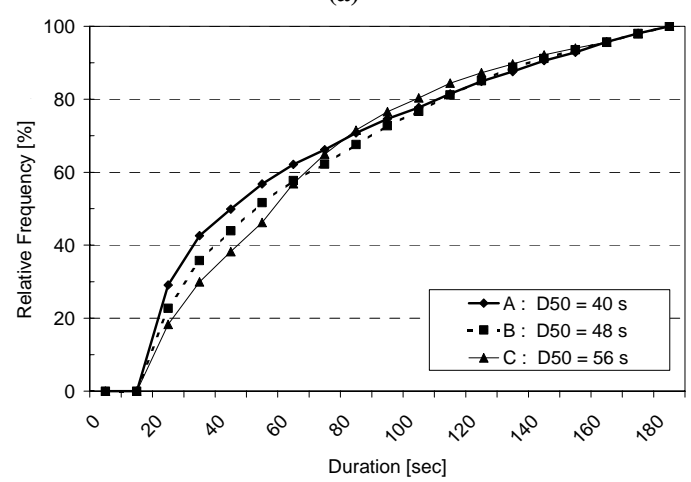

(b)

Fig-6. Relative frequency of short interruptions (durations between $10 \mathrm{~s}$ and 3 min.). (a) Relative frequency histograms. (b) Cumulative frequency curves. 
Figure 6 (a) gives the average number of short interruptions per semester whose durations are indicated in the horizontal scale (the interval $10 \mathrm{~s}-3$ min. was divided into 10 seconds wide classes). Figure 6 (b) gives the cumulative frequency curves.

\section{CONCLUSIONS.}

Long duration interruptions recorded during the last three years, over a sample of locations in the Buenos Aires metropolitan area, were processed to obtain reliability indexes such as the interruption rate $(\lambda)$, the mean time to repair $(r)$ and the unavailability $(Q)$. Table 2 summarizes the obtained results (quantities are mean statistical values).

Table 2. Reliability indexes. Mean values.

\begin{tabular}{|l|c|c|c|}
\hline Utility & A & B & C \\
\hline$\lambda$ [n/location-semester $]$ & 7.17 & 6.19 & 6.94 \\
\hline$r$ [h/interruption] & 1.39 & 1.09 & 1.46 \\
\hline$Q$ [h/location-semester] & 8.74 & 5.70 & 7.69 \\
\hline
\end{tabular}

For microinterruptions, occurrence rates (mean values) were obtained for each Utility. These values may be considered as a Power Quality index. Obtained results are summarized in Table 3 , where quantities are mean statistical values.

Table 3. Microinterruption rates. Mean values [microinterruptions/semester].

\begin{tabular}{|l|c|c|c|}
\hline Utility & A & B & C \\
\hline Microinterruption 1 & - & - & - \\
\hline Microinterruption 2 & 5.4 & 4.9 & 6.6 \\
\hline Microinterruption 3 & 6.2 & 6.3 & 7.4 \\
\hline
\end{tabular}

As a complementary study, duration modal values have been obtained for microinterruptions whose durations are comprised between $10 \mathrm{~s}$ and $3 \mathrm{~min}$. It has been found that the distribution of the interruption duration do not show peculiarities.

It is important to denote that the results of this work were obtained from data recorded at locations qualified as with a low power supply quality. The selection of such locations is because at present the Regulatory Agency (ENRE) gives priority to the validation of the interruptions reported each semester by the Utilities rather than the statistical evaluation of the quality and reliability indexes.

Nevertheless this fact does not invalidate the method presented here to evaluate the system reliability indexes. To obtain the overall network representative values it would suffice to adequate the location selection criteria to assure the sample representativeness.

In a near future, once the validity is assured, the method may be applied, as a statistical evaluator of the global calculus process used by the Utilities to compute the discounts (bonus) due to non supplied energy (NSE), as it was originally conceived.

\section{REFERENCES.}

1. Contratos de Concesión de los Servicios de Distribución Eléctrica del Área Metropolitana de Buenos Aires, Argentina. Subanexo 4.

2. Ente Nacional Regulador de la Electricidad (ENRE). "Resolución 527/96. Base metodológica para el Control de la Calidad del Servicio Técnico. Etapa 2". Buenos Aires, Argentina, 1996.

3. International Union of Producers and Distributors of Electrical Energy (UNIPEDE). 1991 N. 50.02 and "Application Guide to the European Standard EN 50160". January, 1995.

4. European Standard EN 50160. "Voltage characteristics of electricity supplied by public distribution systems". 1999. 